\title{
Aula de Videojuegos, un proyecto académico
}

\author{
Mónica BARrientos Bueno \\ Universidad de Sevilla \\ mbarrientos@us.es \\ José Luis NAVARRETE CARDERO \\ Universidad de Sevilla \\ lnavarrete@us.es
}

Recibido: 24/07/2012

Aceptado: 17/10/2012

\begin{abstract}
Resumen
Aula de Videojuegos es un proyecto académico multidisciplinar de investigación, formación y desarrollo de la Facultad de Comunicación de la Universidad de Sevilla, en el que también participan la de Bellas Artes y la Escuela Técnica Superior de Ingeniería Informática. Las variadas actividades desempeñadas se expanden más allá de los límites característicos de un proyecto de investigación al uso; tienen por propósito la formación práctica de sus integrantes en destrezas relacionadas con el desarrollo del videojuego, así como la realización de diversas investigaciones, las cuales serán canalizadas principalmente tanto en una serie de libros con la editorial Síntesis, como en la propia revista científica del proyecto: $L i$ fePlay. Todo ello pretende reactivar el sector industrial del videojuego en el entorno andaluz y convertir el Aula en referente internacional de investigación.
\end{abstract}

Palabras clave: Videojuego, investigación, formación, desarrollo, Aula de Videojuegos.

\section{Videogame classroom, an academic project}

\begin{abstract}
At the University of Seville, the Faculty of Communication features Videogame classroom as an academic multidisciplinary project for research, teaching and development, where Faculty of Fine Arts and School of Computer Engineering also participate. The variety of activities performed are expanded beyond the typical limits of a research project, in that they employed solely: teach skills related to the development of a videogame and carry out various types of research which will be published by Sintesis Publishers and by LifePlay, our own academic journal. This approach strives to reactivate the Andalusian videogame industry and convert this project into an international role model in videogame research. Keywords: Videogame, research, teaching, development, Videogame classroom.
\end{abstract}

\section{Referencia normalizada}

BARRIENTOS BUENO, Mónica y NAVARRETE CARDERO, José Luis (2012): “Aula de Videojuegos, un proyecto académico". Estudios sobre el mensaje periodístico. Vol. 18, núm. especial octubre, págs.: 111-119. Madrid, Servicio de Publicaciones de la Universidad Complutense.

Sumario: 1. Introducción. 2. Metodología y objetivos. 3. Materialización del proyecto Aula de Videojuegos; 3.1. Sede y personal; 3.2. Áreas y funciones; 3.2.1. Área de Formación; 3.2.2. Área de Investigación; 3.2.3. Área de Desarrollo; 3.2.4. Área de Documentación; 3.2.5. Área de Gestión y Comunicación. 4. Conclusiones. 5. Referencias bibliográficas.

\section{Introducción}

El mundo del videojuego, en los últimos tiempos, ha ido ganando relevancia en los estudios, investigaciones y espacios académicos, reflejo de la importancia creciente de este nuevo medio, el único surgido en la era informática. Incluso para los estudiosos más profanos y menos avezados en el tema salta a la vista que los videojuegos no son un reducto lúdico infantil, son muchos los adultos de países industrializados que jue- 
gan, que invierten su tiempo en Assassin's Creed o Dishonored, de forma que ocupa un volumen relevante de horas de tiempo de ocio maduro. Éste es un aspecto que ha convertido su industria, en el ámbito de las producciones culturales del sector de la informática y las comunicaciones, en la más rentable, con cifras que incluso llegan a sobrepasar a la cinematográfica en las comparativas de niveles de inversiones y rentabilidad de sus productos (Newman, 2009: 3-5).

La intensa labor llevada a cabo desde los trabajos académicos y publicaciones que han empezado a tomar el videojuego como un elemento de estudio dentro del marco de la industria y la cultura audiovisuales (Montagnana, 2008; Newman, 2008 y 2009, Carrillo y Sebastián, 2010), inciden en el hecho de que tiene más vinculación con la producción y la difusión audiovisual que con la industria del juguete o con el campo del ocio despreocupado ${ }^{1}$. Consecuencia lógica de ello es que se haya convertido en objeto de análisis audiovisual, considerado así como una novedosa forma de expresión artística que reúne música, guión, dibujo, diseño e informática. "Un arte al menos tan complejo como las formas clásicas del audiovisual" (Levis, 1997: 38), con estudios donde se parte, entre otros aspectos, de que "tanto los relatos como los juegos poseen estructuras narrativas y propician vivencias narrativas en los individuos; pero, mientras que en el relato el individuo 'vive' una historia ajena en la que no participa, con el juego el individuo 'vive' una historia propia en cuyo desarrollo y resolución participa activamente" (Ruiz, 2008: 19). En definitiva, los videojuegos "se han hecho un hueco entre el gran público gracias a la posibilidad de sumergirse en la interactividad total" (Montagnana, 2008: 9). Aunque si queremos trazar un panorama certero de las investigaciones llevadas a cabo alrededor del videojuego, no podemos olvidar que un gran porcentaje de los trabajos y proyectos de investigación emprendidos se centran en los efectos psicológicos de la violencia de algunos títulos en jóvenes y adolescentes (Estallo, 1997), los particulares hábitos de consumo (Levis, 1997) y sus aplicaciones didácticas (Del Moral et al., 2012; Monjelat y Méndez, 2012; Revuelta y Guerra, 2012; Del Castillo et al., 2012).

En el entorno español destacan iniciativas en el ámbito universitario como el Observatorio del Videojuego y la Animación de la Universidad Europea de Madrid (observatoriovideojuegos.wordpress.com), puesto en marcha en 2003 y que ha desarrollado varias investigaciones sobre nuevas tendencias y alternativas en el sector del videojuego, los hábitos y preferencias de las mujeres que juegan, los usos más frecuentes entre los jugadores mayores de 35 años, entre otros. A ello se suman proyectos de investigación con financiación oficial, como "Análisis de los flujos de transferencia de conocimiento entre los sistemas educativos superiores y la industria del videojuego" (2012-2014), llevado adelante por el equipo de Investigación en Tecnologías Aplicadas a la Comunicación Audiovisual (Itaca) de la Universitat Jaume I de Castellón (www.labcap.uji.es). Recientemente, en 2010, se ha creado en Bilbao un centro de alto rendimiento en programación y diseño de videojuegos gracias al acuerdo

1 Como los últimos años demuestran, esta sentencia de Levis: "los videojuegos rara vez han merecido la atención de los investigadores en comunicación social” (1997: 22) ya no es descriptiva del panorama actual. 
entre DigiPen Institute of Technology y la Cámara de Comercio de Bilbao (www.digipen.es), que promueve esencialmente la formación al más alto nivel profesional.

El Máster en Guión, Narrativa y Creatividad Audiovisual, del Departamento de Comunicación Audiovisual, Publicidad y Literatura de la Universidad de Sevilla, con ya dos ediciones a sus espaldas, acoge entre sus módulos formativos el guión de videojuegos como propuesta curricular para complementar la formación del alumnado con las nuevas formas de ficción interactivas que presenta la industria del ocio audiovisual. En este entorno surge, en 2012, Aula de Videojuegos en la Facultad de Comunicación ${ }^{2}$ como un proyecto académico multidisciplinar que no sólo abarca la investigación del videojuego como discurso narrativo y lúdico, sino también la formación del alumnado en su diseño y el desarrollo íntegro de juegos. Por ello trasciende los límites convencionales de un proyecto estrictamente de investigación para abrirse a otros aspectos que amplían sus inmediatos horizontes, como se ha señalado y en las siguientes páginas se va a exponer.

\section{Metodología y objetivos}

La metodología empleada para la organización estructural y funcional del Aula de Videojuegos ha sido esencialmente intuitiva: parte de los objetivos generales expuestos en el punto anterior que definen las líneas en las que trabajar: investigación, formación y desarrollo. Así éstas se iban a constituir en las áreas esenciales de trabajo, las que se hacen más visibles de cara al exterior por la difusión y publicación de los trabajos de investigación, por los cursos especializados y de formación abierta y, finalmente, por la implementación de una aplicación de videojuego íntegramente creada y desarrollada por miembros del Aula, junto a otra en colaboración con una empresa del sector. Las necesidades han hecho de obligada creación otras dos más: documentación, por un lado, y gestión y comunicación, por otro. Otro instrumento metodológico al que se ha recurrido es la propia experiencia de los coordinadores de las distintas secciones que integran el proyecto.

El objetivo principal es fomentar un espacio multidisciplinar erigido sobre las diversas perspectivas teórico-prácticas concitadas en los últimos años alrededor del videojuego. Nuestros intereses combinan la praxis y el desarrollo de juegos con la reflexión crítica y el análisis de uno de los modos de expresión más apasionante de todos los tiempos. Como profesionales de la educación, dentro del marco tecnófilo en el que nos movemos, no podemos obviar la formación del alumnado en disciplinas y materias con un calado creciente en impacto en el mundo empresarial.

El Aula de Videojuegos, a través de la Facultad de Comunicación y la Universidad de Sevilla, pretende sembrar la semilla con la que poder dar el salto hacia el establecimiento de unas bases educativas y formativas que, en unos años, dé frutos en forma de reimpulso del mercado de trabajo en este sector en auge, revertir en riqueza para toda la sociedad y redefinir el modelo económico de Andalucía.

2 Su puesta en marcha, a iniciativa de su director y creador, el profesor José Luis Navarrete, se aprueba el 27 de marzo de 2012 por parte de la Junta de la Facultad de Comunicación de la Universidad de Sevilla. 


\section{Materialización del proyecto Aula de Videojuegos}

\subsection{Sede y personal}

Tiene su sede física, infraestructura imprescindible para el transcurso de sus actividades, en el espacio denominado "Aula de Videojuegos", en la primera planta de la Facultad de Comunicación de la Hispalense. Se ha presentado al ámbito universitario en la Facultad de Comunicación, con la presencia del reputado especialista Jaume Esteve, en la Escuela Técnica Superior de Ingeniería Informática y en la Facultad de Bellas Artes.

El Aula de Videojuegos, compuesta de varias áreas de formato participativo, tiene su gran patrimonio en el capital humano, el cual lo conforman profesores de las facultades de Comunicación, Bellas Artes y la Escuela Técnica Superior de Ingeniería Informática junto a egresados y alumnos de grado y máster de diversas especialidades, además de profesionales del videojuego del ámbito andaluz.

\section{2. Áreas y funciones}

Las secciones en las que se organiza el Aula de Videojuegos son cinco, como se ha señalado anteriormente, cada una de ellas con al menos un coordinador-profesor universitario al frente encargado de la supervisión:

1. Área de Formación. Su objetivo principal es la formación del alumnado en diversas disciplinas relacionadas con el videojuego.

2. Área de Investigación. A través de las investigaciones que se desarrollarán en su seno pretende convertir al Aula en un referente internacional en game studies.

3. Área de Desarrollo. Busca llegar al desarrollo completo de una idea propia de videojuego y otra compartida con una empresa del sector.

4. Área de Documentación. Su labor consiste en desarrollar herramientas útiles para investigadores y empresarios del videojuego.

5. Área de Gestión y Comunicación. Diseña estrategias de comunicación y busca formas con las que dar respuesta a las necesidades del Aula.

La coordinación entre las áreas es un aspecto capital, de manera que no se definen ni funcionan como espacios estancos sino que los conocimientos y el trabajo circulan de unas a otras para lograr que el funcionamiento del Aula de Videojuegos sea fluido. Así se obtiene el máximo rendimiento a las labores desarrolladas y que el trabajo de unas áreas revierta en beneficio de otras.

\subsection{1. Área de Formación}

En los pocos meses de funcionamiento del Aula de Videojuegos, este área se ha convertido en una de las vías principales por la que cualquier persona o alumno de la Universidad de Sevilla conoce el proyecto y se integra en él. Acerca directamente el proyecto al público con reuniones y encuentros abiertos. Sus áreas de actuación son dos jornadas: las interaula y las sesiones de open gaming.

En las interaula cualquier persona interesada en compartir sus conocimientos presenta una exposición en el Aula, abriendo posteriormente un turno de debate. La actividad propone un interesante feed-back así como el propio hecho de compartir saberes y competencias, favoreciendo el enriquecimiento recíproco. Entre las sesio- 
nes ya realizadas y proyectadas se encuentran las dedicadas a tratar aspectos industriales (Cómo funciona una empresa de videojuegos), históricos (Los autores más importantes del videojuego, La edad de oro del software español), de desarrollo (Programación para no programadores), actualidad (El quinteto de desarrolladoras más relevantes hoy), diseño y guión (Introducción al análisis del personaje de videojuego, Cómo hacer crítica de un videojuego, Bases del guión de un videojuego).

Las open gaming también están abiertas al público; son jornadas dedicadas exclusivamente a jugar bajo el criterio marcado por el área de investigación, de manera que las experiencias sirvan para el desarrollo de estudios sobre recepción, modalidades de juego a distintos niveles, etc. que estén en curso.

El Área de Formación también desarrolla e imparte cursos especializados. Recientemente se ha llevado a cabo la primera edición de 'Introducción a la teoría, al guión y al diseño de videojuegos' (a través del Centro de Formación Permanente de la Universidad de Sevilla), enfocado a dar una visión introductoria a las tres principales actividades profesionales surgidas en la industria del videojuego, mostrando al alumno el funcionamiento práctico y reflexivo del proceso creativo originado por este nuevo medio lúdico y cultural y, al mismo tiempo, poner de relieve el marco teórico y crítico que ha ido generando como discurso audiovisual. El plantel formativo se complementa con talleres de formación interna a solicitud de las necesidades del Área de Desarrollo.

\subsection{2. Área de Investigación}

Pretende alcanzar un equilibrio entre la teoría y la práctica, lo que implica que su actividad está en diálogo directo con el Área de Desarrollo. Sus objetivos se orientan al estudio teórico, la producción de aportaciones a una praxis del videojuego desde frentes cualitativos y cuantitativos con el fin de establecer una base sólida para los diversos proyectos que se lleven a cabo.

El Área tiene una sección de asesoramiento para Tesis Fin de Máster y Tesis Doctorales que tengan como objeto el videojuego, además del propio despliegue de las investigaciones que se propongan desde otras secciones, especialmente la de Desarrollo.

Otro de los proyectos en marcha es la edición y desarrollo de varias colecciones de volúmenes en colaboración con la Editorial Síntesis. Fruto del trabajo de investigación del Aula se pretende conseguir publicaciones referentes de primer nivel en el ámbito académico. Se organiza en varias series, las cuales contarán con materiales interactivos y contenido digital audiovisual producidos por el Área de Desarrollo:

- Cultura. Enfocada a desvelar las relaciones entre el videojuego y otras disciplinas como la Psicología, el Psicoanálisis, la Sociología, la Narratología o la Ludología.

- Diseño y creación de videojuegos. Se va a profundizar en el funcionamiento y valor de los procesos técnicos y creativos que participan en la gestación de un videojuego. La especificidad del género hace necesaria, además, la inclusión de algunos estudios sobre la teoría del videojuego. Actualmente, la línea de investigación más prolífica se asienta en el establecimiento de su ontología, ya sea como objeto lúdico o como discurso narrativo. 
- Autores y maestros del videojuego. A pesar de que la noción de autoría es una de las más discutidas por la teoría del videojuego, es ineludible apuntar algunos nombres referentes que han modelado el medio, marcando los hitos que han forjado su historia. Es una serie que tiene vocación de interpretar las obras señeras y desvelar nombres propios que, aunque poco conocidos, han dado creaciones de gran valor.

- Plataformas de videojuegos. Es un recorrido por las consolas y plataformas que han marcado el desarrollo del medio; más que ningún otro elemento el hardware apunta la relación del jugador con el juego y ello lo convierte en un aspecto de gran interés para el conocimiento del videojuego.

- Producción de videojuegos. Serie que se centrará en el análisis y estudio de los tres modelos industriales del videojuego: el americano, el japonés y el europeo.

Al mismo tiempo, se mantiene un blog en El País (http://blogs.elpais.com/aula-devideojuegos/) como espacio de difusión del pensamiento sobre el videojuego desde distintas perspectivas y enfoques (desde las más prácticas a las más teóricas), sin perder el parámetro académico. Permite que el proyecto haga partícipes, tanto a lectores especializados como al gran público, de las reflexiones de cinco firmas fijas de integrantes del Aula. Las entradas se organizan en cinco categorías: crítica y actualidad, culturas del videojuego, España crea, retrospectivas y diario del Aula.

En breve saldrá el primer número de la revista científica del Aula de Videojuegos: LifePlay, donde tendrán cabida ediciones monográficas, miscelánea y reseñas bibliográficas. Se propone convertirse en referente hemerográfico académico internacional en la temática del juego.

\subsection{3. Área de Desarrollo}

De todas las secciones es la que proporciona el carácter más práctico al ir más allá de las fronteras de lo académico y repercutir directamente en la sociedad. Su objetivo es experimentar con los conocimientos presentes en el Aula y desarrollar aplicaciones de carácter educativo y lúdico. Se plantea el desarrollo de un proyecto propio del Aula y otro en colaboración con una empresa externa.

Aquí entran en juego aptitudes de diversas ramas, como la programación, el diseño, el arte y la comunicación audiovisual, por ello se subdivide en tres subáreas que optimicen las sinergias de trabajo de sus miembros, tuteladas por un coordinador:

- Guión y diseño. Es un laboratorio de ideas, guiones, narrativas y diseño de videojuegos.

- Arte. Trabaja sobre la conceptualización del juego: diseño visual de personajes, escenarios, interfaz, texturas en 2D y 3D.

- Programación. Proporciona la cobertura tecnológica, imprescindible para la materialización de un juego realizado de principio a fin por el Aula de Videojuegos.

Las tres subsecciones, en función de sus necesidades, tienen competencias en la demanda de talleres específicos de aprendizaje al Área de Formación. 


\subsection{4. Área de Documentación}

La principal tarea que actualmente desempeña es la confección de una base de datos en castellano consultable en internet, a través de la web del Aula, de todas las empresas españolas del videojuego y sus títulos. En el proyecto colaboran específicamente profesores de la Escuela Técnica Superior de Ingeniería Informática y del área de conocimiento de información y documentación de la Facultad de Comunicación, junto a alumnos de ambos centros.

Junto a la base de datos, el área de documentación creará y mantendrá una ludoteca: una colección de videojuegos que podrán ser empleados tanto para las investigaciones en curso, así como para las sesiones de open gaming y el préstamo interno dentro del ámbito de los miembros que integran el Aula de Videojuegos.

\subsection{5. Área de Gestión y Comunicación}

Supone el nexo con el mundo empresarial y académico, al mismo tiempo que sirve de firme conexión para las distintas áreas del proyecto académico. De todas, es el área más transversal, visibiliza el Aula y canaliza la búsqueda de financiación privada o pública, así como los recursos de naturaleza diversa necesarios para el desempeño de las labores y actividades de las otras secciones. Igualmente tiene adscritas funciones de comunicación y relación con los medios. Otras de sus labores son:

- community management: mantenimiento del sitio web del proyecto (http://institucional.us.es/videojuegos/), del perfil institucional en Facebook (https://www.facebook.com/AulaDeVideojuegos) y de la cuenta en Twitter (@aulavideojuegos). La diversificación de canales de comunicación responde a la necesidad de difundir las actividades del Aula de Videojuegos por distintas vías y llegar a distintos públicos según sus preferencias en redes sociales y de navegación por internet.

- relación con los medios de comunicación, desarrolladoras, blogueros de interés, otras universidades y grupos de investigación. El propósito es que el Aula de Videojuegos esté integrada tanto en el mundo académico y de investigación como en el propio del videojuego (industria, jugadores, etc.), de esta manera se establecerá un diálogo fluido desde varios frentes, de interés tanto para el desarrollo del proyecto en su integridad como de sus actividades.

- creación de material de difusión y promoción, indispensable para que el proyecto Aula de Videojuegos y sus actividades sean conocidas, así como el perfilado de la imagen institucional del proyecto.

\section{Conclusiones}

El estudio académico del videojuego es uno de los yacimientos más prósperos y crecientes en el entorno de la comunicación, siendo posible la multiplicidad de enfoques y puntos de vista. Su importancia industrial y auge como producto cultural, cuya producción es equiparable a la cinematográfica en varios estratos, justifica ya por sí solo el que, en el entorno del Máster en Guión, Narrativa y Creatividad Audiovisual, del Departamento de Comunicación Audiovisual, Publicidad y Literatura de la Universi- 
dad de Sevilla, surgiera la idea del proyecto académico Aula de Videojuegos. Tiene su sede física en la Facultad de Comunicación, ubicada en el Parque Tecnológico de la Isla de la Cartuja; su naturaleza interdisciplinar ha hecho que de forma natural estreche lazos con la Escuela Técnica Superior de Ingeniería Informática y la Facultad de Bellas Artes, de donde proceden algunos de sus coordinadores y parte de los alumnos que la integran.

Aula de Videojuegos es proyecto donde se combinan la reflexión, la investigación y la crítica compara de videojuegos de forma coordinada con trabajos de desarrollo y formación. Reflejo de ello son los diversos proyectos en los que actualmente se encuentra embarcada: una pionera colección editorial con Síntesis, sesiones de aprendizaje, cursos formativos específicos, una revista académica sobre videojuegos (LifePlay), una base de datos de empresas y títulos españoles de libre acceso, el desarrollo íntegro de dos juegos, entre otros.

Lo que comenzó como un pequeño proyecto académico, con un puñado de integrantes, ha ido creciendo con el paso de los meses en capital humano, competencias, destrezas, contactos empresariales, ideas y planes a largo, medio y corto plazo. El tiempo confirmará la trascendencia alcanzada por el proyecto y su impacto a nivel empresarial andaluz y académico nacional e internacional. Simplemente acabamos de comenzar.

\section{Referencias bibliográficas}

CARRILLO MARQUETA, Juan y SEBASTIÁN MORILLAS, Ana (coords., 2010): Marketing Hero. Las herramientas comerciales de los videojuegos. Madrid, ESIC

DEL CASTILlO, Héctor, HERRERO, David, GARCÍA VARELA, Ana Belén, CHECA, Mirian y MONJELAT, Natalia (2012): "Desarrollo de competencias a través de los videojuegos deportivos: alfabetización digital e identidad", en $R e$ vista de Educación a Distancia: http://www.um.es/ead/red/33/delCastillo_et_al.pdf [fecha de consulta: 20 de octubre de 2012]

DEL MORAL PÉREZ, Ma Esther, VILLALUSTRE MARTÍNEZ, Lourdes, YUSTE TOSINA, Rocío y ESNAOLA, Graciela (2012): "Evaluación y diseño de videojuegos: generando objetos de aprendizaje en comunidades de práctica", en Revista de Educación a Distancia: http://www.um.es/ead/red/33/esther_et_al.pdf [fecha de consulta: 20 de octubre de 2012]

ESTALLO MARTÍ, Juan Alberto (1997): "Psicopatología y videojuegos": http://www.ub.edu/personal/videoju.htm [fecha de consulta: 14 de octubre de 2012].

LEVIS, Diego (1997): Los videojuegos, un fenómeno de masas. Qué impacto produce sobre la infancia y la juventud la industria más próspera del audiovisual. Barcelona: Paidós

MONJELAT, Natalia y MÉNDEZ, Laura (2012): "Videojuegos y diversidad: construyendo una comunidad práctica en el aula", en Revista de Educación a Distancia: $\mathrm{http}: / /$ www.um.es/ead/red/33/monjelat_mendez.pdf [fecha de consulta: $20 \mathrm{de}$ octubre de 2012] 
MONTAGNANA, Vincent (2008): Videojuegos. Una nueva forma de cultura. Barcelona, Robin Book

NEWMAN, James (2008): Playing with videogames. London, Routledge Taylor \& Francis Group

NEWMAN, James (2009): Videogames. London, Routledge Taylor \& Francis Group

REVUELTA RODRÍGUEZ, Francisco Ignacio y GUERRA ANTEQUERA, Jorge (2012): “¿Qué aprendo con los videojuegos? Una perspectiva de meta-aprendizaje del jugador", en Revista de Educación a Distancia: http://www.um.es/ead $/$ red/33/revuelta.pdf [fecha de consulta: 20 de octubre de 2012]

RUIZ COLLANTES, F. X. (2008): "Juegos y videojuegos. Formas y vivencias narrativas", en SCOLARI, Carlos A.: L'homo videoludens. Videojocs, textualitat $i$ narrativa interactiva. Vic, Eumo Editorial

\section{Mónica BARRIENTOS BUENO}

Universidad de Sevilla

mbarrientos@us.es

Profesora Ayudante Doctor del Departamento de Comunicación Audiovisual, Publicidad y Literatura de la Universidad de Sevilla

\section{José Luis NAVARRETE CARDERO}

Universidad de Sevilla

lnavarrete@us.es

Profesor Contratado Doctor del Departamento de Comunicación Audiovisual, Publicidad y Literatura de la Universidad de Sevilla

Dirección postal común: Facultad de Comunicación. Avenida Américo Vespucio s/n. 41092 Sevilla 